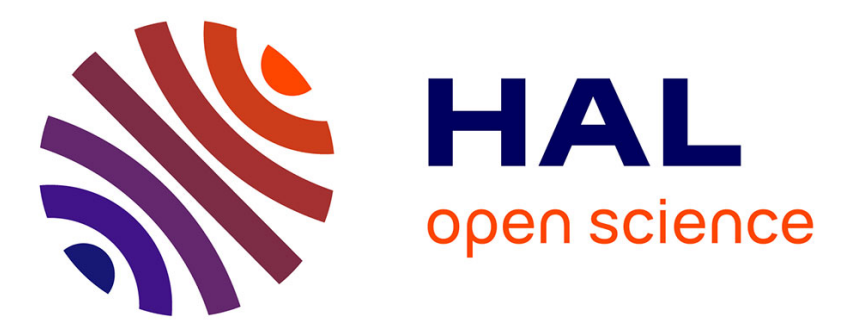

\title{
Volume Integral Formulation Using Face Elements for Electromagnetic Problem Considering Conductors and Dielectrics
}

Jonathan Siau, Gérard Meunier, Olivier Chadebec, Jean-Michel Guichon, Rémy Perrin-Bit

\section{To cite this version:}

Jonathan Siau, Gérard Meunier, Olivier Chadebec, Jean-Michel Guichon, Rémy Perrin-Bit. Volume Integral Formulation Using Face Elements for Electromagnetic Problem Considering Conductors and Dielectrics. IEEE Transactions on Electromagnetic Compatibility, 2016, 58 (5), pp.1587 - 1594. 10.1109/TEMC.2016.2559801 . hal-01385629

\section{HAL Id: hal-01385629 \\ https://hal.science/hal-01385629}

Submitted on 22 Jul 2020

HAL is a multi-disciplinary open access archive for the deposit and dissemination of scientific research documents, whether they are published or not. The documents may come from teaching and research institutions in France or abroad, or from public or private research centers.
L'archive ouverte pluridisciplinaire HAL, est destinée au dépôt et à la diffusion de documents scientifiques de niveau recherche, publiés ou non, émanant des établissements d'enseignement et de recherche français ou étrangers, des laboratoires publics ou privés. 


\title{
Volume Integral Formulation Using Face Elements for Electromagnetic Problem Considering Conductors and Dielectrics
}

\author{
Jonathan Siau, Gérard Meunier, Olivier Chadebec, Jean-Michel Guichon, and Rémy Perrin-Bit
}

\begin{abstract}
A new integral formulation is presented, enabling the computation of resistive, inductive, and capacitive effects considering both conductors and dielectrics in the frequency domain. The considered application allows us to neglect any propagation effects and magnetic materials. In this paper, we will show how to improve the unstructured-partial element equivalent circuit approach to consider dielectric materials, keeping the same benefits. Results obtained with this formulation are compared to results from an industrial finite-element method software and measurements.
\end{abstract}

Index Terms-Dielectric materials, magneto-harmonic, unstructured-PEEC, volume integral equation.

\section{INTRODUCTION}

$\mathbf{F}$ OR solving Maxwell's equations, many numerical methods and formulations can be found depending on the restraint assumptions. Finite-element methods (FEMs) are very popular for solving electromagnetic problems [1]-[3], allowing us to assemble only sparse matrices, so any memory issues can be avoided. On the other hand, integral equation methods [4]-[6] (IEMs) lead to assembling and storing full matrices. In the past, full matrices were really problematic due to the technological limitations. But the main advantage of IEM is that we need to mesh only the active regions, whereas the FEM requires meshing the surrounding air in addition. As a consequence, the IEM should be well suited to studying electromagnetic compatibility (EMC) problems. EMC applications mostly consist in studying distant components (such as wireless charging applications) or compact devices (such as printed circuit boards) and both have a large amount of air. So, using the FEM necessitates having a dense mesh in the air region around the devices, leading to practical issues (such as meshing difficulties or insufficient memory

Manuscript received September 11, 2015; revised January 11, 2016 and April 11, 2016; accepted April 12, 2016.

J. Siau is with the Cedrat S.A., Meylan 38246, France, the University Grenoble Alpes, G2Elab, Grenoble 38000, France, and also with the C.N.R.S., G2Elab, Grenoble 38000, France (e-mail: jonathan.siau@ cedrat.com).

G. Meunier, O. Chadebec, and J.-M. Guichon are with the University Grenoble Alpes, G2Elab, Grenoble 38000, France, and also with the C.N.R.S., G2Elab, Grenoble 38000, France (e-mail: gerard.meunier@g2elab.grenoble-inp. fr; olivier.chadebec@g2elab.grenoble-inp.fr; jean-michel.guichon@g2elab. grenoble-inp.fr).

R. Perrin-Bit is with the Cedrat S.A., Meylan 38246, France (e-mail: remy. perrinbit@cedrat.com). available). Also, the IEM enables us to compute the electromagnetic fields everywhere in space using only the active regions, so far interactions can be easily studied. Moreover, nowadays the IEM has regained interest due to new technologies and compression techniques. Many compression techniques exist, like clustering techniques such as the FMM [7] or the $\mathrm{H}$-matrices [8].

The applications of many electromagnetic formulations are often academic and their use for industrial purposes can be really a handful. A difficulty in modeling industrial applications in the electrical engineering domain is adding external electric components (like supply sources). A method has been proposed by Ruehli based on integral equations to solve efficiently electromagnetic problems coupled with an external circuit: the partial element equivalent circuit (PEEC) [9]. It consists in transforming a meshed electromagnetic device into its equivalent circuit made of lumped R-L-C elements and sources, so the coupling with the external circuit is natural. The classical PEEC was limited to structured meshes, but this limitation has recently been overcome by a Nonorthogonal PEEC formulation [10] or by using face elements [11], [12] for general meshes. This major improvement enables the treatment of more complex geometries and devices [13]. In practice, the PEEC method is mostly used to model conductors and resistive, inductive and capacitive effects [14]-[16]. A few previous publications have considered dielectric materials [18], [19], which have limitations. For instance, in [19] the magneto-quasi-static and electrostatic problems are solved separately, so the phenomena are not strongly coupled. Moreover, the user has to define macroelements manually (which are a group of elements of the mesh) for the electrostatic solution, which may add some inaccuracy to the electrical phenomena.

The contribution of this paper is to propose a formulation which extends the PEEC method to take into account resistive, inductive, and capacitive effects in the presence of conductors and dielectric materials. Only one solution is needed here to solve the whole problem and the capacitive regions are supported by the mesh, so we have overcome the limitations of the previous literature. Moreover, the only assumptions here are that we neglect propagation effects (low frequencies) and that there is no magnetic material. Therefore, we will be using the freespace Green's function which does not take into account any delay. However, these assumptions are usual for low-frequency applications.

In the first section, we present the proposed formulation, starting from Maxwell's equations. Then, a practical formulation is 
introduced to overcome a limitation of the last one, in which a few assumptions will be stated. Last, the validation of the formulation is done with two examples. In the first one, we will compare the results of an industrial FEM software with the practical formulation, and in the second, the results are compared to real measurements. Note that the formulations will be expressed in the frequency domain, but they can be easily written in the time domain.

\section{FORMULATION}

Let us start by defining the following regions: $\Omega_{J}$ and $\Omega_{D}$ contain, respectively, the conductors and the dielectrics, and $\Omega=\Omega_{J} \cup \Omega_{D}$. Their borders are defined as $\Gamma=\partial \Omega, \Gamma_{J}=$ $\partial \Omega_{J}$, and $\Gamma_{D}=\partial \Omega_{D}$.

\section{A. Maxwell's Equations}

In a problem with conductors, dielectrics and without any magnetic material, we have the following constitutive laws:

$$
\begin{cases}\mathbf{J}=\sigma \mathbf{E} & \text { in } \Omega_{J}, \\ \mathbf{D}=\epsilon \mathbf{E}=\epsilon_{0} \mathbf{E}+\mathbf{P} & \text { in } \Omega_{D}, \\ \mathbf{B}=\mu_{0} \mathbf{H} & \end{cases}
$$

where $\mathbf{J}$ is the current density, $\mathbf{E}$ the electric field, $\sigma$ the conductivity, $\mathbf{D}$ the displacement current, $\mathbf{P}$ the polarization density, $\mathbf{B}$ the magnetic induction, $\epsilon$ the permittivity, and $\mathbf{H}$ the electromagnetic field. These quantities are expressed in SI units. According to Maxwell's equations, we get

$$
\begin{aligned}
\operatorname{rot} \mathbf{E} & =-j \omega \mathbf{B}=-\mu_{0} j \omega \mathbf{H} \\
\operatorname{rot} \mathbf{H} & =\mathbf{J}+j \omega \mathbf{D}=(\mathbf{J}+j \omega \mathbf{P})+\epsilon_{0} j \omega \mathbf{E}
\end{aligned}
$$

with $j$ the imaginary unit, $\omega=2 \pi f$, and $f$ the frequency. As in a vacuum, we can also write the potentials in the presence of dielectrics using the Lorentz gauge, as follows:

$$
\begin{aligned}
\mathbf{A} & =\frac{\mu_{0}}{4 \pi} \int_{\Omega} \frac{\mathbf{J}+j \omega \mathbf{P}}{r} \mathrm{~d} \Omega \\
j \omega V & =\frac{1}{4 \pi \epsilon_{0}} \int_{\Omega}(\mathbf{J}+j \omega \mathbf{P}) \nabla\left(\frac{1}{r}\right) \mathrm{d} \Omega
\end{aligned}
$$

where $\mathbf{A}$ and $V$ are the magnetic vector potential and the scalar electric potential, respectively, and $r$ is the distance between the testing and the integration points. We can note that only the $j \omega \mathbf{P}$ term has been added from the original expression.

A previous formulation was presented in [12] to take into account resistive and inductive effects in the presence of conductors. The choice was made to use the current density $\mathbf{J}$ as the unknown. Moreover, as the current density's normal component is preserved between two elements, it seems natural to use the face element discretization. This also permits overcoming the structured mesh limitation of the classical PEEC.

Now, we want to take into account capacitive effects and dielectric materials, so we have to use another unknown having the same conservative property in order to keep the same benefits. We choose the total current density $\mathbf{J}_{\text {tot }}$ as the new unknown:

$$
\mathbf{J}_{\text {tot }}=\mathbf{J}+j \omega \mathbf{D}=\sigma^{*} \mathbf{E}
$$

with $\sigma^{*}=\sigma+j \omega \epsilon$, which can be seen as a complex conductivity varying in the matter. $\mathbf{J}_{\text {tot }}$ obviously keeps the same benefits in the conductors, and in the dielectric $\mathbf{J}_{\text {tot }}=j \omega \mathbf{D}$ verifies the same property.

Thus, this integral equation formulation consists in matching the electrical constitutive law (6) everywhere in the materials (conductors and dielectrics) with

$$
\mathbf{E}=-j \omega \mathbf{A}-\nabla V
$$

resulting in

$$
\frac{\mathbf{J}_{\mathrm{tot}}}{\sigma^{*}}=-j \omega \mathbf{A}-\nabla V
$$

To express the potential based on $\mathbf{J}_{\text {tot }}$, we use the second constitutive law and (6) to write

$$
\mathbf{J}_{\mathrm{tot}}=\mathbf{J}+j \omega \mathbf{P}+j \omega \epsilon_{0} \mathbf{E}
$$

so we can deduce

$$
\mathbf{J}+j \omega \mathbf{P}=\frac{\sigma^{*}-j \omega \epsilon_{0}}{\sigma^{*}} \mathbf{J}_{\mathrm{tot}}
$$

And by substituting (10) into (4), we can write (8) as

$$
\frac{\mathbf{J}_{\mathrm{tot}}}{\sigma^{*}}+j \omega \frac{\mu_{0}}{4 \pi} \int_{\Omega} \frac{\sigma^{*}-j \omega \epsilon_{0}}{\sigma^{*}} \frac{\mathbf{J}_{\mathrm{tot}}}{r} \mathrm{~d} \Omega=-\nabla V .
$$

\section{B. Discretization}

To be able to treat unstructured meshes, we use the same test and trial functions as in [12]. So, we discretize $\mathbf{J}_{\text {tot }}$ using the face elements $\mathbf{w}_{j}$ as follows:

$$
\mathbf{J}_{\mathrm{tot}}=\sum_{k} \mathbf{w}_{k} \mathbf{I}_{k}
$$

with $\mathbf{I}_{j}$ the current flowing through the face $j$.

Finally, applying a standard Galerkin projection to (11) with the same functions leads to the matrix system

$$
\begin{aligned}
\left(\left[\mathbf{R}_{\mathbf{t}}\right]+j \omega\left[\mathbf{L}_{\mathbf{t}}\right]\right)\{\mathbf{I}\} & =\left\{\delta V_{\text {int }}\right\} \\
{\left[\mathbf{R}_{\mathbf{t}}\right]_{i, j} } & =\int_{\Omega} \frac{\mathbf{w}_{i} \mathbf{w}_{j}}{\sigma^{*}} \mathrm{~d} \Omega \\
{\left[\mathbf{L}_{\mathbf{t}}\right]_{i, j} } & =j \omega \int_{\Omega} \mathbf{w}_{i} \int_{\Omega} \frac{\sigma^{*}-j \omega \epsilon_{0}}{\sigma^{*}} \frac{\mathbf{w}_{j}}{r} \mathrm{~d} \Omega \mathrm{d} \Omega \\
\left(\delta V_{\text {int }}\right)_{i} & =-\int_{\Omega} \mathbf{w}_{i} \nabla V \mathrm{~d} \Omega
\end{aligned}
$$

with $\left[\mathbf{R}_{\mathbf{t}}\right]$ a sparse matrix which represents the resistive effect in the conductor and capacitive effects in the dielectrics, and $\left[\mathbf{L}_{\mathbf{t}}\right]$ is the inductive dense matrix. We can see an example of an equivalent circuit representation inFig. 2 of the mesh in Fig. 1. Note that Fig. 2 shows only the self-element contribution (not the mutuals), but the complete circuit can be easily constructed using [14, Fig. 1]. We are going to investigate the meaning of $\delta V_{\text {int }}$, and we start by using the Divergence Theorem:

$$
-\left(\delta V_{\text {int }}\right)_{i}=\int_{\Omega} \mathbf{w}_{i} \nabla V \mathrm{~d} \Omega=\int_{\Gamma}\left(\mathbf{w}_{i} \cdot \mathbf{n}\right) V \mathrm{~d} \Gamma-\int_{\Omega}\left(\boldsymbol{\nabla} \cdot \mathbf{w}_{i}\right) V \mathrm{~d} \Omega .
$$




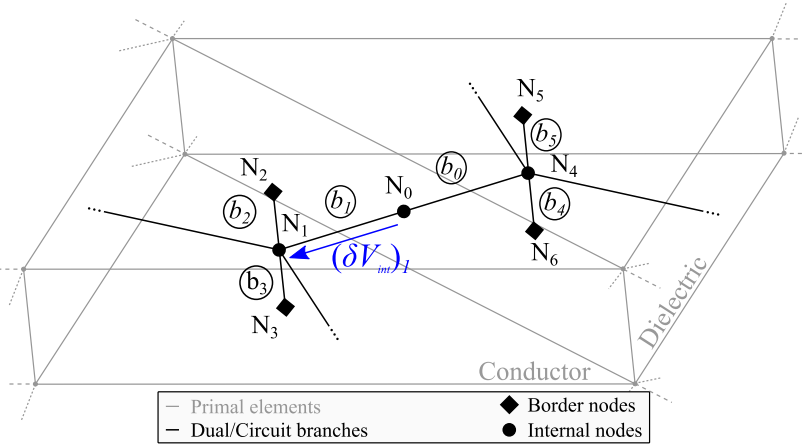

Fig. 1. Example of a topology at the border between a conductor and a dielectric. Each face of the primal mesh corresponds to a dual branch and the black (circuit) nodes correspond to volume elements and border faces.

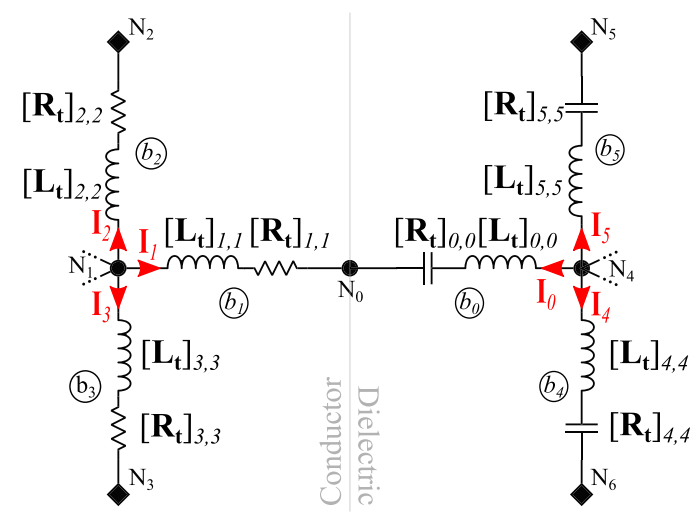

Fig. 2. Simplified equivalent circuit (the mutuals are hidden) of the geometry shown in Fig. 1.

Considering an inner face, we have

$$
\left(\delta V_{\text {int }}\right)_{i}=\int_{\Omega}\left(\nabla \cdot \mathbf{w}_{i}\right) V \mathrm{~d} \Omega
$$

and we know that the support of the face element $\mathbf{w}_{i}$ is shared by two elements ( $e_{1}$ and $e_{2}$, see Fig. 1$)$, so we have

$$
\left(\delta V_{\text {int }}\right)_{i}=\int_{e_{1}} \frac{V}{\operatorname{Vol}\left(e_{1}\right)} \mathrm{d} \Omega-\int_{e_{2}} \frac{V}{\operatorname{Vol}\left(e_{2}\right)} \mathrm{d} \Omega
$$

knowing that $\boldsymbol{\nabla} \cdot \mathbf{w}_{i}=\operatorname{Vol}\left(e_{i}\right)^{-1}$, with $\operatorname{Vol}\left(e_{i}\right)$ giving the volume of the element $e_{i}$. We can see that (19) represents the difference of averaged potential between two inner elements. So, from a circuit approach, we can assimilate this as the applied voltages.

Let's consider a face $j$ on the border. Its support is only on one element ( $e_{1}$ for example), so (17) gives

$$
\left(\delta V_{\text {int }}\right)_{j}=\int_{\Gamma}\left(\mathbf{w}_{j} \cdot \mathbf{n}\right) V \mathrm{~d} \Gamma-\int_{e_{1}} \frac{V}{\operatorname{Vol}\left(e_{1}\right)} \mathrm{d} \Omega
$$

and knowing that $\left(\mathbf{w}_{j} \cdot \mathbf{n}\right)=S_{j}^{-1}$, with $S_{j}$ the surface of the face $j$, we have

$$
\left(\delta V_{\text {int }}\right)_{j}=\int_{\Gamma_{j}} \frac{V}{S_{j}} \mathrm{~d} \Gamma-\int_{e_{1}} \frac{V}{\operatorname{Vol}\left(e_{1}\right)} \mathrm{d} \Omega
$$

also representing the potential difference between a border face and its inner support.
So, the right-hand side vector is the potential difference between the dual mesh branches (see Fig. 1), where the dual mesh represents the equivalent electrical circuit.

Finally, (13) corresponds to the incomplete circuit equations $(\mathbf{U}=\mathbf{Z I})$ : the circuit is not closed (see Fig. 1). So, we will have to add new branches on the border faces, and to express their contributions. For example, we have to add four capacitive branches in Fig. 2 (linking the nodes $\mathrm{N}_{2}, \mathrm{~N}_{3}, \mathrm{~N}_{5}$, and $\mathrm{N}_{6}$ to the common node $\infty$ ) in order to close the circuit. To complete the equations, we have to express the difference of potential between the border faces, so we introduce the common node $\infty$ and

$$
\left(\delta V_{\mathrm{ext}}\right)_{j}=V_{\Gamma_{j}}-V_{\infty}
$$

with $V_{\Gamma_{j}}$ and $V_{\infty}$ the potential of the border face $j$ and of the common node $\infty$, respectively. Actually, we have already expressed $V_{\Gamma_{j}}$ in the previous case:

$$
V_{\Gamma_{j}}=\int_{\Gamma_{j}} \frac{V}{S_{j}} \mathrm{~d} \Gamma_{j}
$$

We suppose that the node $\infty$ has null potential $\left(V_{\infty}=0\right)$, which leads to

$$
\left(\delta V_{\text {ext }}\right)_{j}=V_{\Gamma_{j}} .
$$

Last, we can use (5) to get

$$
\left(\delta V_{\text {ext }}\right)_{j}=\frac{1}{4 \pi \epsilon_{0}} \int_{\Gamma} \frac{1}{S_{j}} \int_{\Omega} \frac{\sigma^{*}-j \omega \epsilon_{0}}{j \omega \sigma^{*}} \mathbf{J}_{\text {tot }} \nabla\left(\frac{1}{r}\right) \mathrm{d} \Omega \mathrm{d} \Gamma
$$

and using the same discretization procedure, we can write

$$
\left\{\delta V_{\mathrm{ext}}\right\}=\frac{1}{j \omega}\left[\mathbf{P}_{\mathbf{t}}\right]\{\mathbf{I}\}
$$

with

$$
\left[\mathbf{P}_{\mathbf{t}}\right]_{i, j}=\frac{1}{4 \pi \epsilon_{0}} \int_{\Gamma} \frac{1}{S_{i}} \int_{\Omega} \frac{\sigma^{*}-j \omega \epsilon_{0}}{\sigma^{*}} \mathbf{w}_{j} \nabla\left(\frac{1}{r}\right) \mathrm{d} \Omega \mathrm{d} \Gamma .
$$

Here, we have managed to express the difference of potential on the border faces, which can be assimilated as capacitive effects. Last, we have to solve the equation:

$$
\left(\left[\mathbf{R}_{\mathbf{t}}\right]+j \omega\left[\mathbf{L}_{\mathbf{t}}\right]+\frac{1}{j \omega}\left[\mathbf{P}_{\mathbf{t}}\right]\right)\{\mathbf{I}\}=\{\delta V\}
$$

with $\delta V=\delta V_{\text {int }}+\delta V_{\text {ext }}$ the difference of potential of all the branches.

We saw that (28) actually corresponds to a circuit equation, so we can solve it using a circuit solver. The main advantage is that this permits ensuring the conservation of current (Kirchhoff's first law) in the problem. The solver circuit we chose uses the independent loop search approach, instead of the modified nodal approach (usually picked in the literature [20]). This choice was made to minimize of the number of unknowns. However, the final equation to solve is

$$
[\mathbf{M}]\left(\left[\mathbf{R}_{\mathbf{t}}\right]+j \omega\left[\mathbf{L}_{\mathbf{t}}\right]+\frac{1}{j \omega}\left[\mathbf{P}_{\mathbf{t}}\right]\right)[\mathbf{M}]^{T} \mathbf{I}_{m}=0
$$

with $[\mathbf{M}]$ the change of basis matrix from the branches to the independent-loop basis and $\mathbf{I}_{m}$ the loop current vector. 
At this point, we have presented a general approach to include dielectric materials and the capacitive effects. This formulation needs matrices with a dependency on the frequency ( $\sigma^{*}$ and $\frac{\sigma^{*}-j \omega \epsilon_{0}}{\sigma^{*}}$ for instance). This necessitates assembling each matrix once for each frequency we want to study. So, for a frequency study, this is a critical disadvantage, and necessitates using a more practical formulation.

\section{PRACTICAL FORMULATION}

In this section, we will present a more practical formulation using the same approach, but making a few more assumptions to overcome the last critical point.

First, we assume that

$$
\sigma \gg \omega \epsilon_{0}
$$

so the previous unknown $\mathbf{J}_{\text {tot }}$ becomes

$$
\widetilde{\mathbf{J}}_{\text {tot }}=\left\{\begin{array}{ll}
\mathbf{J} & \text { in } \Omega_{J} \\
j \omega \mathbf{D} & \text { in } \Omega_{D}
\end{array} .\right.
$$

Following the same protocol as in Section II, we write the Ampère-Maxwell equation (7) as

$$
\mathbf{E}+j \omega \frac{\mu_{0}}{4 \pi} \int_{\Omega} \frac{\mathbf{J}+j \omega \mathbf{P}}{r} \mathrm{~d} \Omega=-\nabla V
$$

and we can also write

$$
\mathbf{J}+j \omega \mathbf{P}= \begin{cases}\mathbf{J}=\widetilde{\mathbf{J}}_{\text {tot }} & \text { in } \Omega_{J} \\ j \omega \mathbf{P}=\frac{\epsilon-\epsilon_{0}}{\epsilon} \widetilde{\mathbf{J}}_{\text {tot }} & \text { in } \Omega_{D}\end{cases}
$$

using (1). In consequence, (31) can be rewritten as

$$
\mathbf{E}+j \omega \frac{\mu_{0}}{4 \pi}\left(\int_{\Omega_{J}} \frac{\widetilde{\mathbf{J}}_{\mathrm{tot}}}{r} \mathrm{~d} \Omega+\int_{\Omega_{D}} \frac{\epsilon-\epsilon_{0}}{\epsilon} \frac{\widetilde{\mathbf{J}}_{\mathrm{tot}}}{r} \mathrm{~d} \Omega\right)=-\nabla V .
$$

Now, we can apply the Galerkin projection with the interpolation functions $\mathbf{w}_{i}$ :

$$
\begin{aligned}
& \int_{\Omega} \mathbf{w}_{i} \mathbf{E} \mathrm{d} \Omega+j \omega \frac{\mu_{0}}{4 \pi} \int_{\Omega} \mathbf{w}_{i}\left(\int_{\Omega_{J}} \frac{\widetilde{\mathbf{J}}_{\mathrm{tot}}}{r} \mathrm{~d} \Omega\right. \\
& \left.+\int_{\Omega_{D}} \frac{\epsilon-\epsilon_{0}}{\epsilon} \frac{\widetilde{\mathbf{J}}_{\text {tot }}}{r} \mathrm{~d} \Omega\right) \mathrm{d} \Omega=-\int_{\Omega} \mathbf{w}_{i} \cdot \nabla V \mathrm{~d} \Omega .
\end{aligned}
$$

And we can easily see from the first two constitutive laws that

$$
\mathbf{E}= \begin{cases}\frac{\widetilde{\mathbf{J}}_{\mathrm{tot}}}{\sigma} & \text { in } \Omega_{J} \\ \frac{\widetilde{\mathbf{J}}_{\mathrm{tot}}}{j \omega \epsilon} & \text { in } \Omega_{D}\end{cases}
$$

and, by using the same discretization as (12), we finally have

$$
\left([\widetilde{\mathbf{R}}]+j \omega[\widetilde{\mathbf{L}}]+\frac{1}{j \omega}[\widetilde{\mathbf{C}}]\right)\{\mathbf{I}\}=\left\{\delta V_{\text {int }}\right\}
$$

with

$$
\begin{aligned}
{[\widetilde{\mathbf{R}}]_{i, j} } & =\int_{\Omega_{J}} \frac{\mathbf{w}_{i} \mathbf{w}_{j}}{\sigma} \mathrm{d} \Omega \\
{[\widetilde{\mathbf{C}}]_{i, j} } & =\int_{\Omega_{D}} \frac{\mathbf{w}_{i} \mathbf{w}_{j}}{\epsilon} \mathrm{d} \Omega \\
{[\widetilde{\mathbf{L}}]_{i, j} } & =\frac{\mu_{0}}{4 \pi} \int_{\Omega} \mathbf{w}_{i}\left(\int_{\Omega_{J}} \frac{\mathbf{w}_{j}}{r} \mathrm{~d} \Omega+\int_{\Omega_{D}} \frac{\epsilon-\epsilon_{0}}{\epsilon} \frac{\mathbf{w}_{j}}{r} \mathrm{~d} \Omega\right) \mathrm{d} \Omega
\end{aligned}
$$

We can see that we have one more matrix than for (13) $([\widetilde{\mathbf{C}}])$. In fact, the previous matrix $\left[\mathbf{R}_{\mathbf{t}}\right]$ has been simplified to $[\widetilde{\mathbf{R}}]+\frac{1}{j \omega}[\widetilde{\mathbf{C}}]$. So, even if we have added a matrix, the actual amount of memory needed has been divided by 2 , because $\left[\mathbf{R}_{\mathbf{t}}\right]$ is complex while $[\widetilde{\mathbf{R}}]$ and $[\widetilde{\mathbf{C}}]$ are real matrices. We can make the same remark about $[\widetilde{\mathbf{L}}]$ and $\left[\mathbf{L}_{\mathbf{t}}\right]$, so the whole storage has been globally divided by 2 .

At this point, we still have to complete the circuit equation to close the equivalent circuit, and to take into account the capacitive effects. The protocol is exactly the same, so let's begin with (24):

$$
\left(\delta V_{\mathrm{ext}}\right)_{j}=V_{\Gamma_{j}}=\int_{\Gamma_{j}} \frac{V}{S_{j}} \mathrm{~d} \Gamma_{j}
$$

Using (32) in (5) leads to

$$
\begin{aligned}
\left(\delta V_{\text {ext }}\right)_{j}= & \frac{1}{4 \pi \epsilon_{0}} \frac{1}{j \omega} \int_{\Gamma} \frac{1}{S_{j}}\left(\int_{\Omega_{J}} \widetilde{\mathbf{J}}_{\text {tot }} \nabla \frac{1}{r} \mathrm{~d} \Omega\right. \\
& \left.+\int_{\Omega_{D}} \frac{\epsilon-\epsilon_{0}}{\epsilon} \widetilde{\mathbf{J}}_{\text {tot }} \nabla \frac{1}{r} \mathrm{~d} \Omega\right) \mathrm{d} \Gamma .
\end{aligned}
$$

In this case, we can simplify the first twofold integral:

$$
\int_{\Gamma} \frac{1}{S_{i}} \int_{\Omega_{J}} \widetilde{\mathbf{J}}_{\mathrm{tot}} \nabla \frac{1}{r} \mathrm{~d} \Omega \mathrm{d} \Gamma=\int_{\Gamma} \frac{1}{S_{i}} \int_{\Gamma_{J}}\left(\widetilde{\mathbf{J}}_{\mathrm{tot}} \cdot \mathbf{n}\right) \frac{1}{r} \mathrm{~d} \Gamma \mathrm{d} \Gamma
$$

using the Divergence Theorem and knowing that $\operatorname{div} \widetilde{\mathbf{J}}_{\text {tot }}=0$. In addition, assuming that the dielectrics are linear (which implies $\operatorname{div} \mathbf{P}=0$ in $\Omega_{D}$ ), we can reapply the Divergence Theorem to simplify the second twofold integral

$$
\begin{aligned}
& \int_{\Gamma} \frac{1}{S_{j}} \int_{\Omega_{D}} \frac{\epsilon-\epsilon_{0}}{\epsilon} \widetilde{\mathbf{J}}_{\text {tot }} \nabla \frac{1}{r} \mathrm{~d} \Omega \mathrm{d} \Gamma \\
= & \int_{\Gamma} \frac{1}{S_{j}} \int_{\Gamma_{D}} \frac{\epsilon-\epsilon_{0}}{\epsilon}\left(\widetilde{\mathbf{J}}_{\text {tot }} \cdot \mathbf{n}\right) \frac{1}{r} \mathrm{~d} \Gamma \mathrm{d} \Gamma .
\end{aligned}
$$

Now, we use the same discretization on $\widetilde{\mathbf{J}}_{\text {tot }}$ and we can write

$$
\left\{\delta V_{\mathrm{ext}}\right\}=\frac{1}{j \omega}[\widetilde{\mathbf{P}}]\{\mathbf{I}\}
$$

with

$$
\begin{aligned}
{[\widetilde{\mathbf{P}}]_{i, j}=} & \frac{1}{4 \pi \epsilon_{0}} \int_{\Gamma} \frac{1}{S_{i}}\left(\int_{\Gamma_{J}} \frac{1}{S_{j}} \frac{1}{r} \mathrm{~d} \Gamma\right. \\
& \left.+\int_{\Gamma_{D}} \frac{\epsilon-\epsilon_{0}}{\epsilon} \frac{1}{S_{j}} \frac{1}{r} \mathrm{~d} \Gamma\right) \mathrm{d} \Gamma .
\end{aligned}
$$

Here, we have managed to complete the circuit equations with a matrix considering only boundaries. This matrix is much smaller than $\left[\mathbf{P}_{\mathbf{t}}\right]$, so it requires less memory and can be assembled faster. 


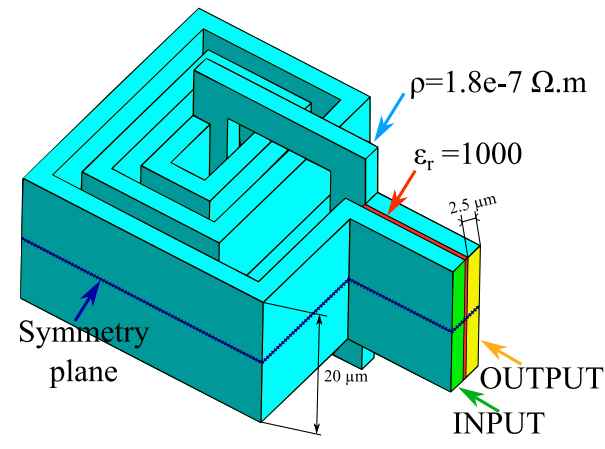

Fig. 3. Studied device: Microcoil with three spires.

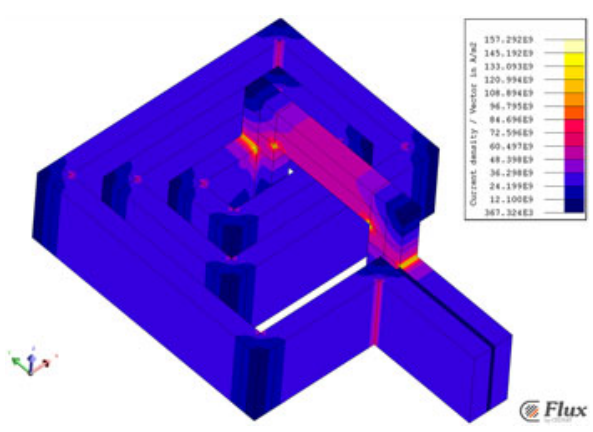

Fig. 4. $\left|\widetilde{\mathbf{J}}_{\text {tot }}\right|$ isovalues at $100 \mathrm{MHz}$ computed using the FEM.

At the end, we have the next equation

$$
\left([\widetilde{\mathbf{R}}]+j \omega[\widetilde{\mathbf{L}}]+\frac{1}{j \omega}([\widetilde{\mathbf{C}}]+[\widetilde{\mathbf{P}}])\right)\{\mathbf{I}\}=\{\delta V\}
$$

to solve. We can note that the matrices do not have any dependency on the frequency, so we have overcome the last critical point. Moreover, we managed to save more than the half of the memory required before. And also, we note that the matrices are purely real, so, using real-assembly codes permits lowering the assembly time.

We also note that the full matrices $([\widetilde{\mathbf{L}}]$ and $[\widetilde{\mathbf{P}}])$ are classical in the theory of integral equations and can be compressed. So, this could allow us to solve bigger problems.

\section{VALIDATION}

The validation of the practical formulation is done comparing the impedances obtained for two examples: a microcoil and a real device. The first example will compare our results to those from an industrial software, and the second will compare our results against measurements.

\section{A. Case 1: Microcoil}

The considered microcoil (see Fig. 3) is made of three conductive spires with a dielectric between the I/O. This validation is done comparing the impedances from the formulation and an industrial finite element software: Flux 3D [21]. Flux3D takes advantage of the symmetry plane on $X Y$, while our integral code cannot. So, the results will show only a half of the device on Figs. 4 and 5, even though the practical formulation used the entire mesh.

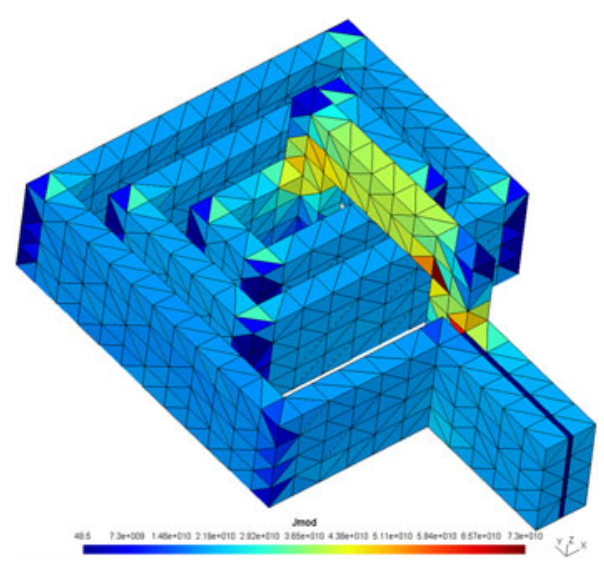

Fig. 5. $\quad\left|\widetilde{\mathbf{J}}_{\text {tot }}\right|$ isovalues at $100 \mathrm{MHz}$ using the "practical formulation."

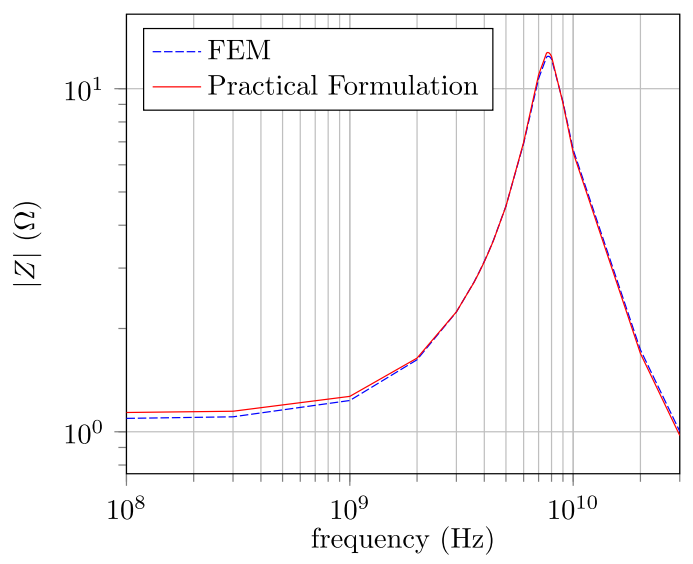

Fig. 6. Impedance modulus versus frequency. In blue (dashed): The reference (FEM), in red: the simulation results.

For a matter of ease, the Flux3D uses a structured mesh for the active regions (conductor and dielectric) composed of 9579 hexahedra and an usual mesh is used in the air with 92049 elements. For the proposed formulation, we used a mesh of 4822 tetrahedra.

The solution of this formulation was done using an FGMRes solver and a Block lower-upper (LU) preconditioner. The FEM solutions used an ICCG solver, so the computational time cannot be fairly compared. The computations have been made using a Dell Precision M4800 with an Intel(R) Core(TM) i7-4800MQ CPU@2.70 GHz and 32Go RAM.

The numerical results are presented in Fig. 6 for the impedance modulus and in Fig. 7 for the impedance phase. In the last two figures, "FEM" stands for the results provided by Flux 3D, which are published in [22].

According to the IEEE standard [23], the comparison and the qualification of the last curves is done using the FSV techniques [24], [25] via the stand-alone software FSV tool [26]. We applied the amplitude difference measure (ADM) and the feature difference measure (FDM) techniques to the impedance modulus curves [see Fig. 8(a) and (b)] and to the impedance phase curves [see Fig. 9(a) and (b)].

We can see in Figs. 6 and 7 that the results of both methods are very similar. This is also supported by the FSV technique 


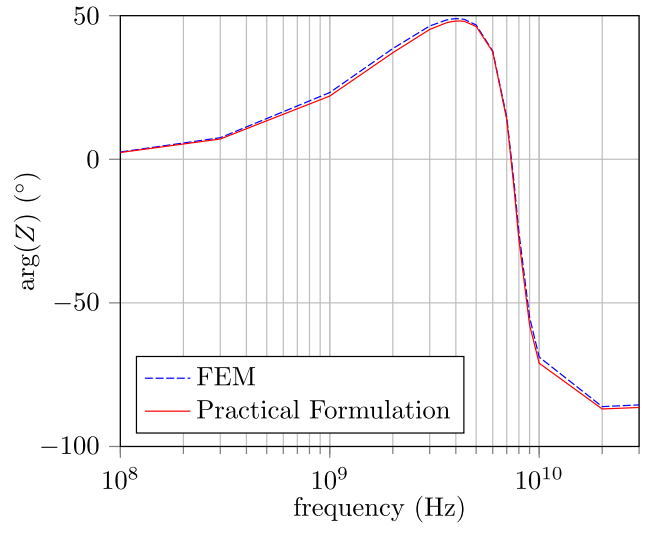

Fig. 7. Impedance phase versus frequency. In blue (dashed): The reference (FEM), in red: the simulation results.

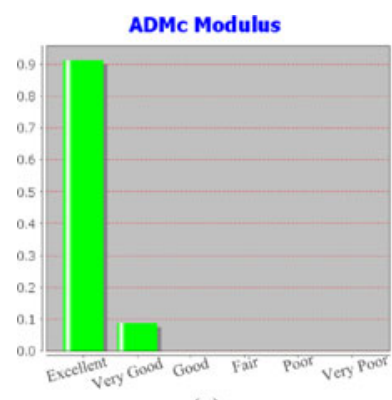

(a)

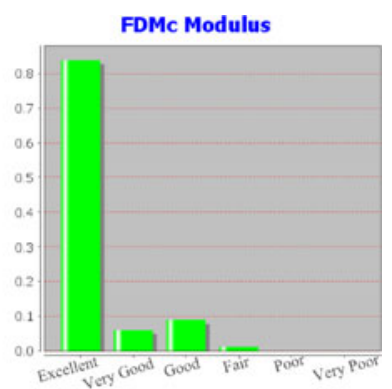

(b)
Fig. 8. (a) Results of the ADM applied to the impedance modulus curves. (b) Results of the FDM applied to the impedance modulus curves.

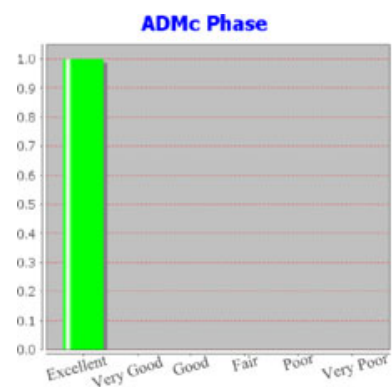

(a)

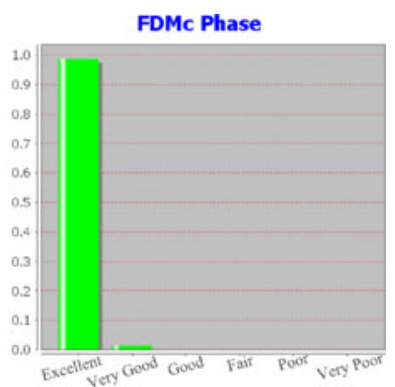

(b)
Fig. 9. (a) Results of the ADM applied to the impedance phase curves. (b) Results of the FDM applied to the impedance phase curves.

results [Figs. 8(a), 8(b), 9(a) and 9(b)] which rates the match of the curves as excellent.

\section{B. Case 2: Real Device}

Let's now consider a real device made of two layers of copper $35 \mu \mathrm{m}$ thick separated by a layer of dielectric FR4 with a thickness of $1.47 \mathrm{~mm}$ (see Fig. 10). Two cases have been considered to do the measurements: The short-circuit case consisting of connecting each layer by a corner (see Fig. 11) and the open-circuit case which consists in leaving the layers without a connexion between them (see Fig. 12). Both cases are considered from 1 to $100 \mathrm{MHz}$.

The same mesh has been used for all the studies and is made of 3210 hexahedra which leads to 16951 faces/degrees of freedom.

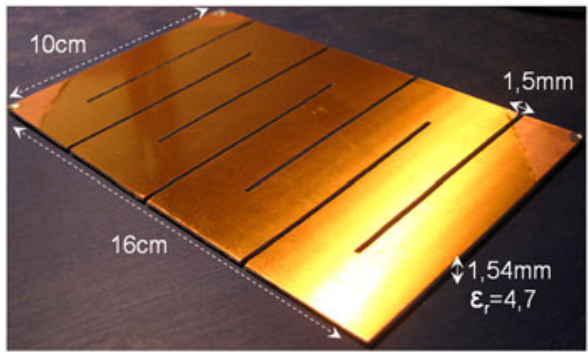

Fig. 10. Picture and dimensions of the studied device.

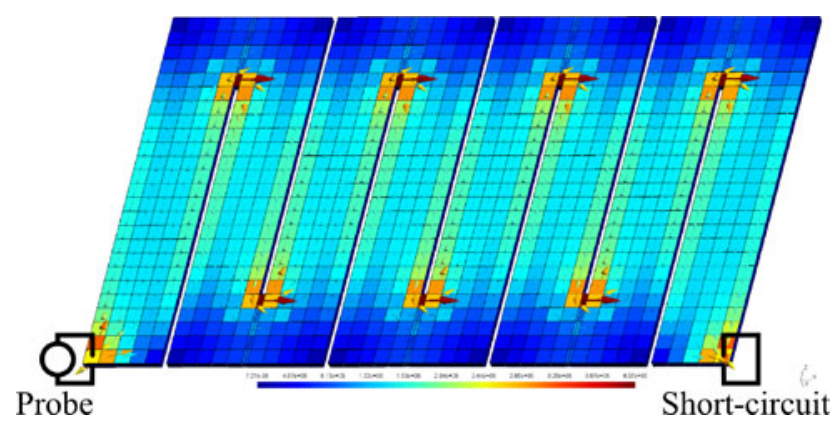

Fig. 11. Current density isovalues of the short-circuit case at $1 \mathrm{MHz}$, with the circuit connexions drawn. The modulus $\left|\widetilde{\mathbf{J}}_{\text {tot }}\right|$ and the real part $\Re\left(\widetilde{\mathbf{J}}_{\text {tot }}\right)$ of the current density are represented by the colored elements and vectors, respectively.

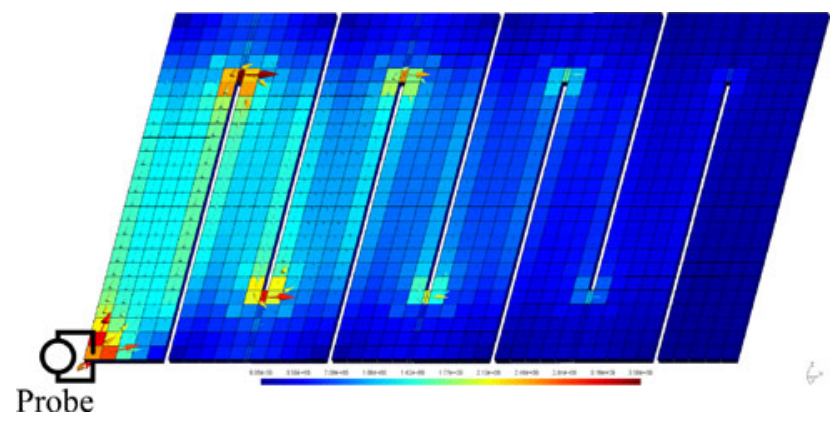

Fig. 12. Current density isovalues of the open-circuit case at $1 \mathrm{MHz}$, with the circuit connexions drawn.

We are using a structured mesh because the geometry is wellsuited and the conductors are very thick, but an unstructured mesh could also have been used. The thickness of each layer is discretized with only one element, because the skin depth is greater than the thickness for the studied frequencies.

The solutions were done using a classical LU factorization, due to the ill-conditioning of the problem. The computations were done on the same laptop as in the previous section. The numerical results are presented in Figs. 13 and 14 for the shortcircuit and the open-circuit case, respectively.

On one hand, both cases show a good accuracy regarding the first peak frequency. On the other hand, the amplitude of the peaks are underestimated. But overall, we see a good match between the curves. To quantify this, we have used the same FSV technique as before (using the FSV Tool). The results are shown in Fig. 15(a) and (b) for the open-circuit case and in Fig. 16(a) and (b) for the short-circuit case. The ADM technique rates both case mostly from good to excellent, whereas the FDM rates them mostly as excellent. 


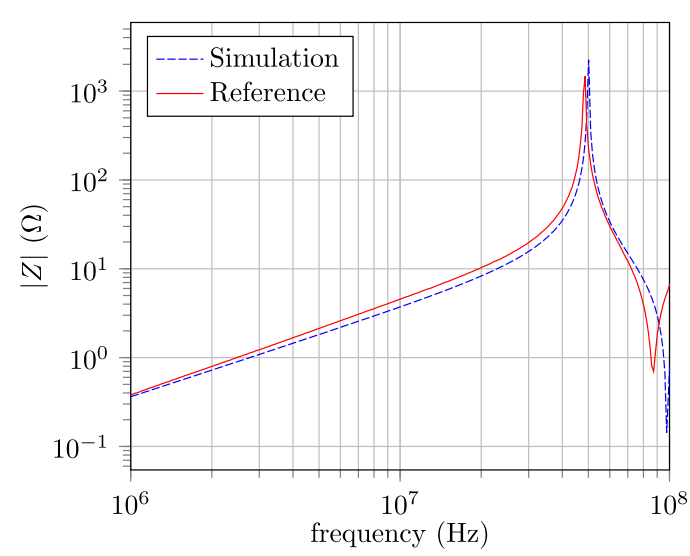

Fig. 13. Impedance modulus versus frequency for the short-circuit case. In blue (dashed): The reference (measurements), in red: the simulation results.

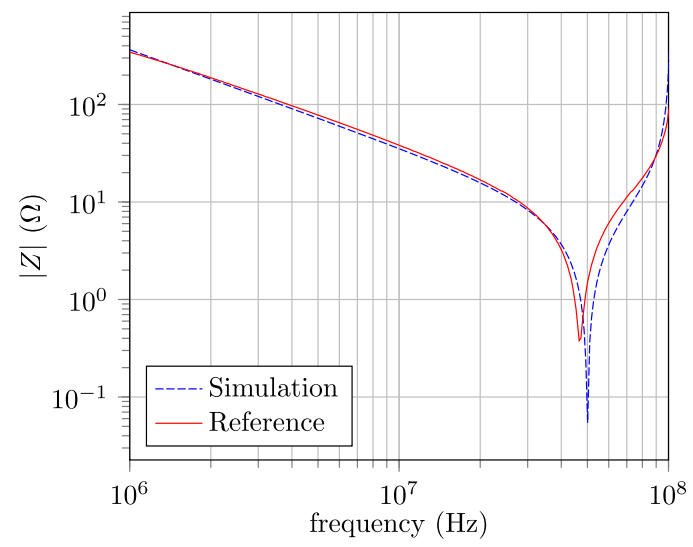

Fig. 14. Impedance modulus versus frequency for the open-circuit case. In blue (dashed): The reference (measurements), in red: the simulation results.

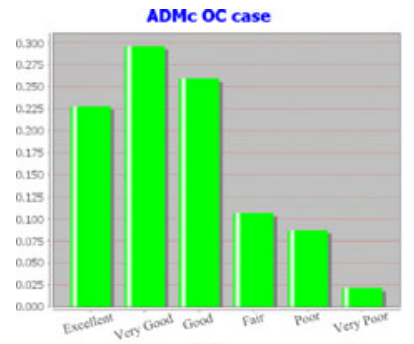

(a)

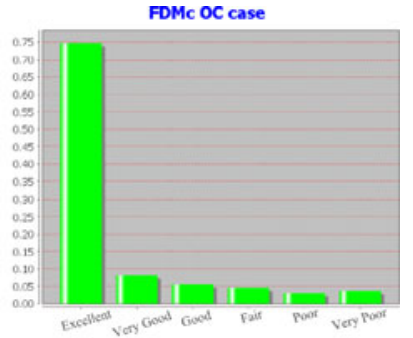

(b)
Fig. 15. (a) Results of the ADM applied to the impedance modulus curves for the open-circuit case. (b) Results of the FDM applied to the impedance modulus curves for the open-circuit case.

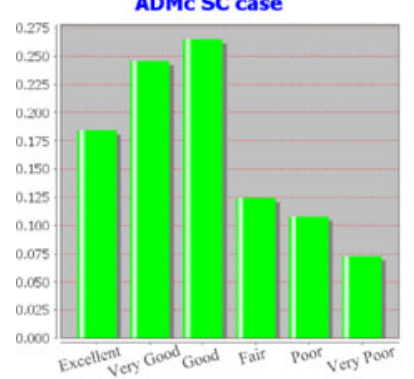

(a)

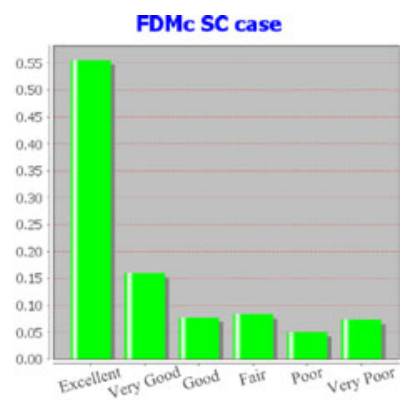

(b)
Fig. 16. (a) Results of the ADM applied to the impedance modulus curves for the short-circuit case. (b) Results of the FDM applied to the impedance modulus curves for the short-circuit case.

\section{CONCLUSION}

We have presented a new formulation to consider resistive, inductive, and capacitive effects in the presence of conductors and dielectrics, with very few assumptions. We pointed out that its generality also spoils its efficiency, due to the frequency dependency of its matrices. So, to solve a problem for multiple frequency one should use the second formulation.

To overcome this dependency, we have presented the practical formulation with a few more assumptions. We have pointed out that these additional restrictions allow us to assemble simpler matrices and to greatly reduce the required storage compared to the general formulation.

The practical formulation has been validated considering two cases. The first consisted in comparing the impedance of a virtual device against an industrial FEM software. The results of both methods show a great match and have been qualified as excellent by the software FSV Tool. The last case consisted in comparing the computed impedances against measurements on a real devices, and the curves show a great match, rated as good to excellent. Further research will compare the proposed formulation to others regarding the computational cost and time.

\section{REFERENCES}

[1] R. Albanese and G. Rubinacci, "Finite element methods for the solution of $3 d$ eddy current problems," Adv. Imag. Electron. Phys., vol. 102, pp. 1-86, 1997.

[2] P. Dular, C. Geuzaine, and W. Legros, "A natural method for coupling magnetodynamic h-formulations and circuit equations," IEEE Trans. Magn., vol. 35, no. 3, pp. 1626-1629, May 1999.

[3] P. Dular, J. Gyselinck, T. Henneron, and F. Piriou, "Dual finite element formulations for lumped reluctances coupling," IEEE Trans. Magn., vol. 41, no. 5, pp. 1396-1399, May 2005.

[4] R. Albanese and G. Rubinacci, "Integral formulation for 3d eddy-current computation using edge elements," IEE Proc. A, Phys. Sci., Meas. Instrum., Manage. Educ.-Rev., vol. 135, no. 7, pp. 457-462, Sep. 1988.

[5] V. Le-Van, G. Meunier, O. Chadebec, and J.-M. Guichon, "A volume integral formulation based on facet elements for nonlinear magnetostatic problems," IEEE Trans. Magn., vol. 51, no. 7, pp. 1-6, Jul. 2015.

[6] G. Rubinacci and A. Tamburrino, "Automatic treatment of multiply connected regions in integral formulations," IEEE Trans. Magn., vol. 46 no. 8, pp. 2791-2794, Aug. 2010.

[7] L. Greengard and V. Rokhlin, "A fast algorithm for particle simulations," J. Comput. Phys., vol. 135, no. 2, pp. 280-292, 1997.

[8] W. Hackbusch, "A sparse matrix arithmetic based on $\mathcal{H}$-matrices. I. Introduction to $\mathcal{H}$-matrices," Comput., vol. 62, no. 2, pp. 89-108, 1999.

[9] A. Ruehli, "Equivalent circuit models for three-dimensional multiconductor systems," IEEE Trans. Microw. Theory Techn., vol. 22, no. 3, pp. 216-221, Mar. 1974

[10] A. Ruehli, G. Antonini, J. Esch, J. Ekman, A. Mayo, and A. Orlandi, "Nonorthogonal PEEC formulation for time- and frequency-domain EM and circuit modeling," IEEE Trans. Electromagn. Compat., vol. 45, no. 2 , pp. 167-176, May 2003.

[11] H. Whitney, Geometric Integration Theory. Princeton, NJ, USA: Princeton Univ. Press, 1957.

[12] T.-T. Nguyen, G. Meunier, J.-M. Guichon, O. Chadebec, and T.-S. Nguyen, "An integral formulation for the computation of 3-d eddy current using facet elements," IEEE Trans. Magn., vol. 50, no. 2, pp. 549-552, Feb. 2014.

[13] M. Bandinelli, "A surface PEEC formulation for high-fidelity analysis of the current return networks in composite aircrafts," IEEE Trans. Electromagn. Compat., vol. 57, no. 5, pp. 1027-1036, Oct. 2015.

[14] F. Freschi, G. Gruosso, and M. Repetto, "Unstructured PEEC formulation by dual discretization," IEEE Microw. Wireless Components Lett., vol. 16 no. 10, pp. 531-533, Oct. 2006.

[15] G. Antonini and D. Romano, "Efficient frequency-domain analysis of PEEC circuits through multiscale compressed decomposition," IEEE Trans. Electromagn. Compat., vol. 56, no. 2, pp. 454-465, Apr. 2014. 
[16] F. Ferranti, M. Nakhla, G. Antonini, T. Dhaene, L. Knockaert, and A. Ruehli, "Multipoint full-wave model order reduction for delayed PEEC models with large delays," IEEE Trans. Electromagn. Compat., vol. 53, no. 4, pp. 959-967, Nov. 2011.

[17] G. Antonini and D. Romano, "An accurate interpolation strategy for fast frequency sweep of partial element equivalent circuit models," IEEE Trans. Electromagn. Compat., vol. 56, no. 3, pp. 653-658, Jun. 2014.

[18] H. Heeb and A. Ruehli, "Three-dimensional interconnect analysis using partial element equivalent circuits," IEEE Trans. Circuits Syst. I: Fundamental Theory Appl., vol. 39, no. 11, pp. 974-982, Nov. 1992.

[19] V. Ardon, J. Aime, O. Chadebec, E. Clavel, and E. Vialardi, "MoM and PEEC method to reach a complete equivalent circuit of a static converter," in Proc. 20th Int. Zurich Symp. Electromagn. Compat., Jan. 2009, pp. 273-276.

[20] F. Freschi, "Fast block-solution of PEEC equations," IEEE Trans. Magn., vol. 49, no. 5, pp. 1753-1756, May 2013.

[21] Flux ${ }^{\circledR} 3 D$ Software, (2016). [Online]. Available: www.cedrat.com

[22] P. Pham Quang, C. Guérin, G. Meunier, and Y. Lambeye, "Validation of a $3 \mathrm{~d}$ decoupled magnetodynamic-electric model by determining the impedance of microcoils," in Volume 34: Computer Field Models of Electromagnetic Devices (Studies in Applied Electromagnetics and Mechanics), S. Wiak and E. Napieralska-Juszczak, Eds., Amsterdam, The Netherlands: IOS Press, Nov. 2010, pp. 599-608.

[23] Standard for Validation of Computational Electromagnetics Computer Modeling and Simulation-Part 1, IEEE Standard P1597, Feb. 2008.

[24] A. Duffy, A. Martin, A. Orlandi, G. Antonini, T. Benson, and M. Woolfson, "Feature selective validation (FSV) for validation of computational electromagnetics (CEM). Part I-The FSV method," IEEE Trans. Electromagn. Compat., vol. 48, no. 3, pp. 449-459, Aug. 2006.

[25] A. Orlandi, A. Duffy, B. Archambeault, G. Antonini, D. Coleby, and S. Connor, "Feature selective validation (FSV) for validation of computational electromagnetics (CEM). Part II-Assessment of FSV performance," IEEE Trans. Electromagn. Compat., vol. 48, no. 3, pp. 460-467, Aug. 2006.

[26] A. Orlandi, (2006). Free stand-alone FSV application. [Online]. Available: http://ing.univaq.it/uaqemc/FSV_4_0_3L/

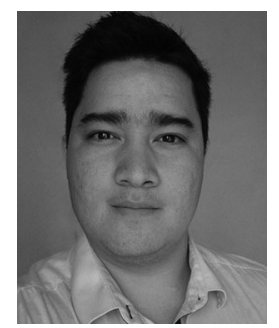

Jonathan SIAU was born in 1989. He received the Master's degree in computational science and applications from the University of Rennes 1, Rennes, France, in 2013. Since 2014, he has been working toward the Ph.D. degree entitled New solvers for 3D power electronics devices simulation, thanks to a collaboration of CEDRAT S.A and the Grenoble Electrical Engineering Laboratory.

His research interests include low-frequency formulations development for the power electronics, their numerical properties, and numerical methods.

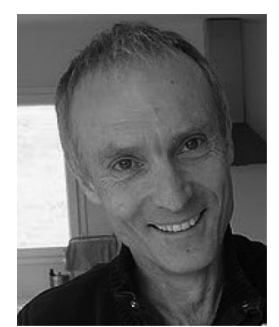

Gérard Meunier was born in 1954. He received the Dipl.-Ing. degree in electrical engineering from the National Polytechnic Institute of Grenoble (INPG), Grenoble, France, in 1977. He received the Ph.D. degree from the INPG in 1981.

He joined the CNRS and the Power Electrical Engineering Laboratory of Grenoble (G2Elab) in 1982 where he is currently a Senior Researcher. His research interest includes numerical modeling of electromagnetic phenomena. In the G2Elab, he was successively responsible of the "Modelling and CAD Team" from 1990 to 1998 and was an Associate Director from 1998 to 2002. He was responsible of the Power Electrical Engineering Doctoral Department from 2002 to 2015 and is currently the Deputy Head of the EEATS Doctoral School, "Communauté Université Grenoble Alpes," Grenoble, France.

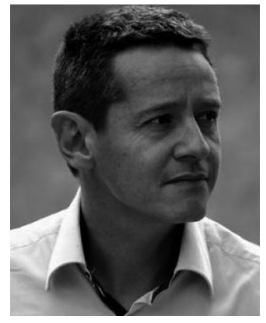

Olivier Chadebec was born in 1973. He received the Diploma in electrical engineering and the Ph.D. degrees from the Grenoble Institute of Technology, Grenoble, France, in 1997 and 2001, respectively.

He is currently the CNRS Senior Researcher (directeur de recherche CNRS) and leads the Models, Methods, and Methodologies Applied to Electrical Engineering Research Group, G2ELab, University of Grenoble, Grenoble, France. He has been a Visiting Scientist at the Technology Centre of the Federal University of Santa Catarina, Brasil, during 2012-2013. His research interests include computational electromagnetism (finite element and integral methods), equivalent magnetic sources identification by solving inverse problems, and low magnetic fields measurements. He has coauthored more than 140 papers published in international journals and conference proceedings.

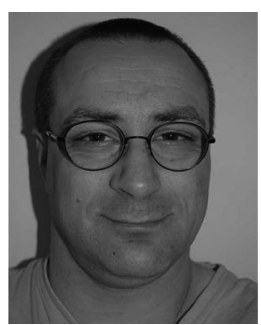

Jean-Michel Guichon was born in 1975. He received the Diploma degree in electrical engineering and the Ph.D. degree from the Grenoble Institute of Technology, Grenoble, France, in 1998 and 2001, respectively.

$\mathrm{He}$ is an Associate Professor at the Universite Grenoble Alpes. He is has been with the Grenoble Electrical Engineering Laboratory, Grenoble, France, since 2003, working in the field of electromagnetic modelization for power electronics. He is focused in the development of numerical tools to compute the electromagnetic field and the electrical behavior in low frequency (without propagation).

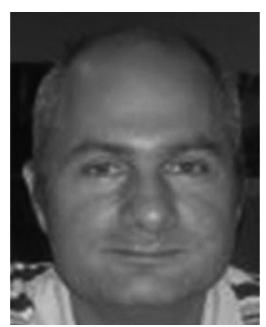

Rémy Perrin-Bit was born in 1967. He received the Diploma in electrical engineering and the Ph.D. degrees from the Ecole Nationale Supérieure d'Ingenieurs Electriciens de Grenoble, Grenoble, France, in 1990, and 1994, respectively.

He is currently System, Physics, and Application Manager of Cedrat, Meylan, France. His activities focus on computational electromagnetism (finite element) in Flux Cedrat software. 